\title{
Diabetic cardiomyopathy in uncomplicated type 2 diabetes is associated with the metabolic syndrome and systemic inflammation
}

Received: 23 February 2005 / Accepted: 16 April 2005 / Published online: 30 June 2005

(C) Springer-Verlag 2005

\begin{abstract}
Abbreviations CRP: C-reactive protein
To the Editor: Even in the absence of hypertension or coronary artery disease, type 2 diabetic patients show myocardial structural and functional changes that are ascribed to so-called diabetic cardiomyopathy $[1,2]$. Changes in cardiac energy metabolism, resulting from altered myocardial substrate supply and utilisation, in association with insulin resistance, may underlie this condition $[3,4]$. In addition to linking insulin resistance, obesity, diabetes and cardiovascular disease, systemic inflammation has been implicated in cardiac functional and structural abnormalities $[5,6]$. We hypothesised that cardiac functional changes in subjects with uncomplicated type 2 diabetes may be associated with a number of features of the metabolic syndrome, as defined by National Cholesterol Education Program guidelines [7], and markers of systemic inflammation. To this purpose, using magnetic resonance imaging, we determined cardiac systolic and diastolic function in 13 asymptomatic normotensive men with recently diagnosed ( $<5$ years), well-controlled type 2 diabetes (mean age $56 \pm 6$ years, $\mathrm{HbA}_{1}$ c $6.1 \pm 1.1 \%$, mean diabetes duration 14 months [range 6-60 months]) and 13 age-matched controls [2].
\end{abstract}

M. Diamant $(\bowtie) \cdot$ R. J. Heine

Department of Endocrinology/Diabetes Centre,

VU University Medical Centre,

De Boelelaan 1117,

P.O. Box 7057, 1007 MB Amsterdam, The Netherlands

e-mail: m.diamant@vumc.nl

Tel.: +31-20-4444444

Fax: $+31-20-4440502$

M. Diamant · J. W. A. Smit

Department of Endocrinology,

Leiden University Medical Centre,

Leiden, The Netherlands

H. J. Lamb · A. de Roos

Department of Radiology,

Leiden University Medical Centre,

Leiden, The Netherlands
Patients had no history or symptoms of cardiovascular disease and all subjects had a normal ECG. Exclusion criteria were microalbuminuria, retinopathy and autonomic neuropathy, as evaluated by Ewing's cardiovascular tests, and the use of insulin or drugs other than oral hypoglycaemic agents. Plasma TNF- $\alpha$ and IL- 6 concentrations and highsensitivity C-reactive protein (CRP) were determined by ELISAs. Because of non-normal distribution, the inflammatory markers were logarithmically transformed. In addition, a systemic inflammation index was calculated as the sum of the logarithms of these inflammatory markers. The protocol conformed with the principles outlined by the Declaration of Helsinki and was approved by the local ethics committee. All subjects gave written informed consent. Comparisons between groups were made by unpaired twotailed Student's $t$-test. Results are presented as means $\pm \mathrm{SD}$ or as medians (interquartile range).

Nine patients and three control subjects satisfied the criteria for the metabolic syndrome. On average, patients had $3.2 \pm 1.0$ features of the metabolic syndrome while control subjects had $1.8 \pm 0.9 \quad(p<0.001)$. Compared with control subjects, patients had higher fasting plasma glucose levels ( $8.2 \pm 1.6$ vs $5.5 \pm 0.4 \mathrm{mmol} / 1, p<0.001)$, higher insulin levels $(120.0 \pm 65.8$ vs $52.7 \pm 18.5 \mathrm{pmol} / 1, p<0.02)$, lower HDL cholesterol $(1.0 \pm 0.3$ vs $1.5 \pm 1.2 \mathrm{mmol} / 1, p<0.001)$ and greater waist circumferences $(99 \pm 5$ vs $94 \pm 7 \mathrm{~cm}, p<0.05)$. Plasma triglyceride levels and blood pressure were similar in the two groups. Of the inflammatory markers, only the mean plasma TNF- $\alpha$ level was higher in patients than in control subjects (median 2.9 [2.6-4.0] vs 2.3 [1.9-3.0] ng/1, $p<0.05$ ); the concentrations of CRP and IL-6 did not differ significantly between the two groups. The systemic inflammation index was higher in patients than in control subjects $(p<0.05)$. Interestingly, when subjects were grouped according to the presence or absence of the metabolic syndrome, irrespective of diabetic state, we observed that all plasma inflammatory markers were elevated in the group that fulfilled the criteria for the metabolic syndrome, as compared with those in the group who did not (TNF- $\alpha 3.1$ 


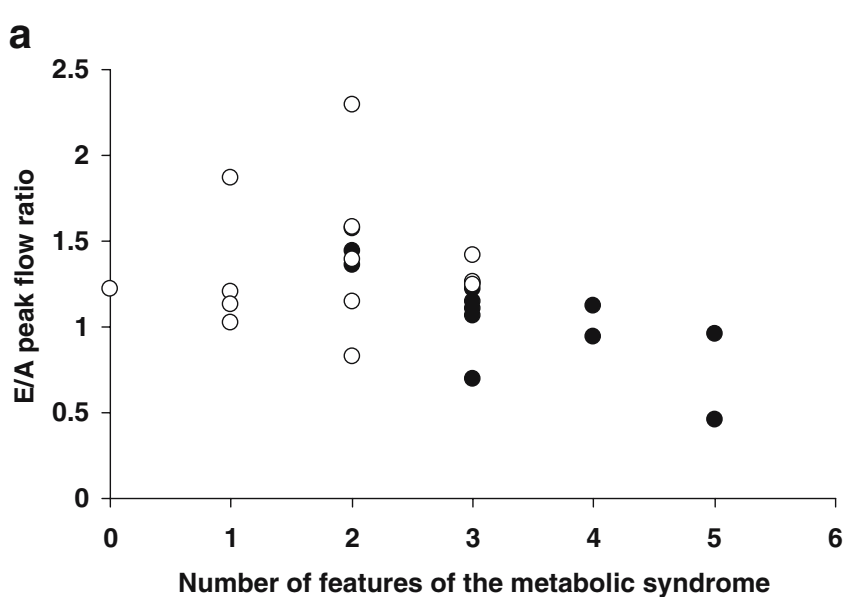

Fig. 1 Scatter plots representing the relationship between E/A peak flow ratio and the number of components of the metabolic syndrome, as defined by National Cholesterol Education Program

[2.5-4.1] vs $2.3[1.8-2.9] \mathrm{ng} / \mathrm{l}, p=0.021 ; \mathrm{CRP} 1.0$ [0.6-3.9] vs $0.5[0.4-1.0] \mathrm{mg} / \mathrm{l}, p=0.034$; IL-6 $1.3[0.8-4.4]$ vs 0.7 $[0.5-0.9] \mathrm{ng} / \mathrm{l}, p=0.013)$. Early to late diastolic peak flow (E/A) ratio, an index of diastolic function, was significantly decreased in patients relative to that in control subjects $(1.1 \pm 0.3$ vs $1.4 \pm 0.4, p<0.02)$. Since no additional measurements were performed to unmask a pseudonormal pattern of ventricular filling, the diastolic functional changes in our patients may have been underestimated. The E/A peak flow ratio in patients, but not in control subjects, was inversely associated with the number of features of the metabolic syndrome $(r=-0.768, p<0.005$; Fig. 1a), and with systemic inflammation $(r=-0.716, p=0.006$; Fig. 1b). In patients, inverse correlations were found between the E/A peak flow ratio and all the inflammatory markers, though the association with IL- 6 was the strongest (TNF- $\alpha r=$ $-0.617, p=0.025$; CRP $r=-0.585, p=0.036$; IL-6 $r=-0.768$, $p=0.002$ ).

The results of this small-scale study indicate that the metabolic syndrome is associated with myocardial diastolic dysfunction in men with well-controlled, uncomplicated type 2 diabetes, and in men with a high level of systemic inflammation. We hypothesize that the abnormalities that characterise the metabolic syndrome may lead to altered energy metabolism in the diabetic heart and adversely affect the energy-demanding molecular mechanisms that are needed to sustain normal diastolic function. The presence of chronic inflammation may further aggravate the compromised diastolic function in these subjects by inducing fibrosis and left ventricular stiffening [8]. However, due to the limited number of observations in this selected population and the cross-sectional nature of the study, we were unable to assess whether (the features of) the metabolic syndrome and inflammation are independent predictors

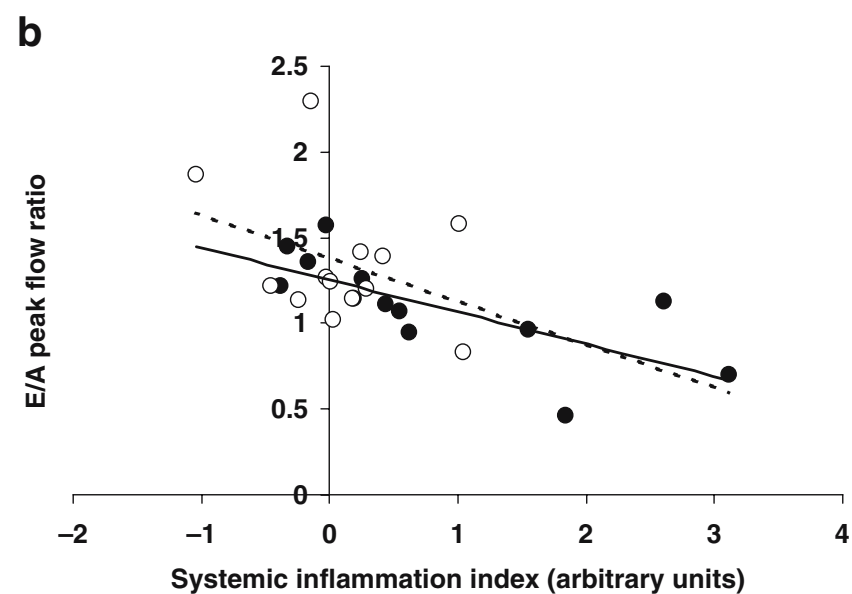

(a), and systemic inflammation (b) in type 2 diabetic patients (black circles, solid line) and healthy controls (white circles, dotted line)

of diastolic dysfunction in type 2 diabetic subjects. Prospective studies should define the predictive value of the metabolic syndrome and systemic inflammation, as well as their possible interaction, during the course of diabetic cardiomyopathy.

\section{References}

1. Bell DS (1995) Diabetic cardiomyopathy. A unique entity or a complication of coronary artery disease? Diabetes Care 18:708-714

2. Diamant M, Lamb HJ, Groeneveld Y et al (2003) Diastolic dysfunction is associated with altered myocardial metabolism in asymptomatic normotensive patients with well-controlled type 2 diabetes. J Am Coll Cardiol 42:328-335

3. Taegtmeyer H, McNulty P, Young ME (2002) Adaptation and maladaptation of the heart in diabetes: Part I. General concepts. Circulation 105:1727-1733

4. Iozzo P, Chareonthaitawee P, Dutka D, Betteridge DJ, Ferrannini E, Camici PG (2002) Independent association of type 2 diabetes and coronary artery disease with myocardial insulin resistance. Diabetes 51:3020-3024

5. Festa A, D'Agostino R Jr, Howard G, Mykkanen L, Tracy RP, Haffner SM (2000) Chronic subclinical inflammation as part of the insulin resistance syndrome: the IRAS study. Circulation 102:42-47

6. Vasan RS, Sullivan LM, Roubenoff R et al (2003) Inflammatory markers and risk of heart failure in elderly subjects without prior myocardial infarction: the Framingham Heart Study. Circulation 107:1486-1491

7. Expert panel on detection, evaluation, and treatment of high blood cholesterol in adults (2001) Executive summary of the third report of the NCEP expert panel on detection, evaluation, and treatment of high blood cholesterol in adults. JAMA 285:2486-2497

8. Nicoletti A, Michel JB (1999) Cardiac fibrosis and inflammation: interaction with hemodynamic and hormonal factors. Cardiovasc Res 41:532-543 